\title{
Comparison of Two Widely Used PTSD-Screening Instruments: Implications for Public Mental Health Planning
}

Kenneth J. Ruggiero, Alyssa A. Rheingold, Heidi S. Resnick, and Dean G. Kilpatrick

Department of Psychiatry and Behavioral Sciences, Medical University of South Carolina, Charleston, $S C$

Sandro Galea

Department of Epidemiology, University of Michigan School of Public Health, Ann Arbor, MI

Epidemiological research serves a critical role in public mental health planning in the aftermath of disasters, particularly via estimation of the mental health burden and potential needs of affected communities. However, different measures are used across studies to assess mental health response, making cross-study comparison difficult. The National Women's Study Posttraumatic Stress Disorder module (NWS-PTSD) and PTSD Checklist (PCL) have been among the most widely used measures of PTSD in postdisaster research. Here, the authors used a sample of 233 New York City-area residents who were administered both the NWS-PTSD and PCL 4 months after the September 11, 2001 terrorist attacks. The PCL yielded higher prevalence estimates at the symptom, cluster, and diagnostic levels. Implications for the interpretation of epidemiological data are discussed.

Posttraumatic stress disorder (PTSD) is the most commonly observed psychiatric disorder in communities affected by disasters and mass violence (Galea, Nandi, \& Vlahov, 2005). As such, assessment of posttraumatic stress reactions is frequently a core feature of postdisaster epidemiological research with population-based samples. However, researchers differ in their approach to the measurement of PTSD as well as their selection of PTSD instruments, thereby limiting cross-study comparability. The use of different assessment instruments is critical because structural differences between assessment methodologies may lead to meaningfully different population-based prevalence estimates and small differences in diagnostic prevalence can produce significant underestimates or overestimates of the post disaster needs of a community. To illustrate, in the 1-2 months following the September 11 terrorist attacks, Schlenger et al. (2002), using the PTSD Checklist (PCL; Weathers, Litz, Herman, Huska, \& Keane, 1993) as an assessment instrument, estimated that $11.2 \%$ of New York City-area residents met criteria for probable PTSD. This estimate was $50 \%$ higher than the $7.5 \%$ prevalence of PTSD estimated by Galea et al. (2002) in a sample of Manhattan residents using the National Women's Study PTSD module (NWS-PTSD; Kilpatrick, Resnick, Saunders, \& Best, 1989) as an assessment instrument. Applying each of these prevalences to the population of over 8 million New York City residents, one would estimate that approximately 900,000 (Schlenger et al.) versus 600,000 (Galea et al.)

This research was funded by National Institutes of Health Grants MH66081, DA14219-S1, and MH066391. Views in this article do not necessarily represent those of the agencies supporting this research.

Correspondence concerning this article should be addressed to: Kenneth J. Ruggiero, Department of Psychiatry and Behavioral Sciences, National Crime Victims Research and Treatment Center, Medical University of South Carolina, P.O. Box 250852, 165 Cannon Street, Charleston, SC 29425. E-mail: ruggierk@musc.edu.

(C) 2006 International Society for Traumatic Stress Studies. Published online in Wiley InterScience (www.interscience.wiley.com) DOI: 10.1002/jts.20141 
persons met criteria for probable PTSD, a difference of 300,000 people. Clearly, a discrepancy of 3.7 percentage points can have significant implications for public mental health planning and resource allocations.

The NWS-PTSD (Kilpatrick et al., 1989) and PCL (Weathers, Litz, Herman, Huska, \& Keane, 1993) are among the most commonly used measures in epidemiological research within the traumatic stress literature. The NWS-PTSD has been used in research on violent crime with nationally representative samples of youth and adults. It has also been used with a wide range of community samples affected by natural and man-made disasters, including the Loma Prieta earthquake, Oakland Hills fire, 1992 Los Angeles Riots, September 11 terrorist attacks, Hurricanes Andrew and Hugo, and the 2004 Florida Hurricanes (e.g., Galea et al., 2002; Garrison et al., 1995; Hanson, Kilpatrick, Freedy, \& Saunders, 1995; Kilpatrick et al., 2003). Similarly, the PCL has been used in a wide range of epidemiologic studies in the traumatic stress literature, including national and community-based studies relating to the September 11 terrorist attacks. In addition, it has been applied in studies with other civilian samples affected by motor vehicle accidents, sexual assault, and cancer, as well as a wide range of combat-exposed samples (e.g., Hoge et al., 2004; Iowa Persian Gulf Study Group, 1997; Schlenger et al., 2002; Schuster et al., 2001). The present study compared these two instruments at the symptom, cluster, and diagnostic level with a sample of New York City-area residents exposed to the terrorist attacks of September 11, 2001. This is intended both to guide researchers who may use either of these instruments in their work and to serve as an exploration of the potential implications of the use of different instruments for public mental health planning.

\section{METHOD}

\section{Participants}

This sample of 233 adults participated in the context of a large household telephone survey of 2,001 New York City-area residents (with an oversampling of Manhattan residents living south of 110th Street) interviewed 4-5 months following the terrorist attacks of September 11, 2001. Demographics of the sample of 233 adults completing both the NWS-PTSD and PCL are as follows. Participants were $54.1 \%$ women and $45.9 \%$ men, with a mean age of 46.0 years $(M d n=44, S D=17.6)$. Racial/ethnic distribution was 54.8\% White, 20.6\% African American, $18.0 \%$ Hispanic, $4.8 \%$ Asian, and $1.8 \%$ other. With regard to participants' level of exposure to the September 11 attacks, 20.6\% lived south of 110th Street on Manhattan (i.e., in close proximity to the World Trade Center site), $26.3 \%$ saw the terrorist attacks in person, and $3.9 \%$ reported having lost their job as a result of the attacks. There were $2.2 \%$ of the participants who reported the death of a relative, $12.2 \%$ the death of a friend, and $24.5 \%$ the death of an acquaintance because of the attacks.

\section{Medsures}

The National Women's Study PTSD module. Developed by Kilpatrick and colleagues (1989), the NWS-PTSD was modified from the Diagnostic Interview Schedule (DIS) used in the National Vietnam Veterans Readjustment Study (Kulka et al., 1990). Major modifications from the DIS included thorough assessment of civilian traumatic event history and the use of follow-up questions to assess symptom content (see the subsection, Structural Differences Between the NWS-PTSD and PCL below). Designed for use by lay interviewers, the NWS-PTSD consists of 20 items that assess Criteria B, C, and D for PTSD as defined in the Diagnostic and Statistical Manual of Mental Disorders, Fourth Edition (DSM-IV; American Psychiatric Association, 1994). The NWS-PTSD assesses the full range of criteria for PTSD, i.e., A through F; however, the present study focuses on components that are comparable to the PCL, that is, Criteria B, C, and D. Research on the NWS-PTSD has provided support for concurrent validity and several forms of reliability (e.g., temporal stability, internal consistency, diagnostic reliability; Kilpatrick et al., 2003; Resnick, Kilpatrick, Dansky, Saunders, \& Best, 1993), and was validated in a field trial against a wellestablished structured diagnostic interview (the Structured 
Clinical Interview for DSM; SCID). In the field trial, the interrater kappa coefficient was .85 for the diagnosis of PTSD, and comparisons between the NWS-PTSD module and the SCID yielded a kappa coefficient of .71 for current and .77 for lifetime PTSD (Kilpatrick et al., 1998). Further, in research with nationally representative samples, the NWS-PTSD has yielded prevalences of traumatic events and PTSD that are similar to those of other national probability studies that carefully assessed both history of life stressors and PTSD, suggesting good construct validity for the NWS PTSD module (e.g., Kessler, Sonnega, Bromet, Hughes, \& Nelson, 1995; Resnick et al., 1993).

The PTSD Checklist. The PCL, developed by Weathers and colleagues (1993), is a 17-item instrument that parallels diagnostic Criteria $\mathrm{B}, \mathrm{C}$, and D for PTSD, as delineated in the DSM-IV. The PCL was designed for use as a selfreport instrument, and is one of only three well-established self-report instruments to correspond closely with each of the 17 DSM-defined PTSD symptoms (Ruggiero, Del Ben, Scotti, \& Rabalais, 2003). Thus, relative to most other available self-report instruments of PTSD, the PCL may yield information that has greater predictive utility on a diagnostic level. Indeed, several psychometric studies have supported the reliability and validity of the PCL with a variety of samples (e.g., combat veterans, college students, motor vehicle accident victims), including diagnostic sensitivity and specificity, internal consistency, test-retest reliability, convergent validity, and discriminant validity (e.g., Blanchard, Jones-Alexander, Buckley, \& Forneris, 1996; Ruggiero et al., 2003; Weathers et al., 1993). Further, the PCL has been adapted for use in interview format in largescale epidemiological studies (e.g., Iowa Persian Gulf Study Group, 1997; Schuster et al., 2001), which is indicative of its support and acceptance in the field.

\section{Structural differences between the NWS-PTSD and} $\boldsymbol{P C L}$. The NWS-PTSD and PCL have a number of similarities. However, they also have structural differences that may relate to prevalence estimates in meaningful ways. First, item content differs in the assessment of certain symptoms. For example, the PCL item "having trouble concentrating" differs somewhat from the corresponding NWS-PTSD item "had trouble concentrating or keeping your mind on what you were doing, even when you tried to concentrate." Second, the NWS-PTSD uses yes/no response options based on past-month presence of a symptom that has had a duration of 2 weeks or longer at some time in the respondent's life, whereas the PCL uses response options ranging from 1 (not at all) to 5 (extremely). Third, the NWS-PTSD is designed to detect symptoms (i.e., occurrence vs. nonoccurrence) irrespective of participants' accompanying distress, whereas the PCL operationalizes symptoms based on their accompanying distress (i.e., "Indicate how much you have been bothered by that problem”). Finally, the NWS-PTSD first assesses symptom occurrence and then uses follow-up questions to identify stressor event(s) to which symptoms are connected (e.g., "What were the nightmares about?"), whereas the PCL specifically instructs participants to endorse symptoms that are linked to a particular event without the use of follow-up questions.

\section{Procedure}

Participants were contacted using random-digit dial methodology. Trained interviewers, guided by a computerassisted telephone interview system, conducted all interviews. Interviews were highly structured, were available in English and Spanish, and were approximately 35 minutes in length. The NWS-PTSD was administered to all participants in the larger study, 233 of whom were also administered the PCL to facilitate comparisons between the NWS-PTSD and PCL. These 233 participants were a randomly selected subset of participants without children under age 18 (participants with children were administered a separate module in its place). The NWS-PTSD consistently was administered prior to the PCL-these modules were not adjacent to one another in the interview. Symptoms for both instruments were assessed for the 30-day period prior to interview. The Institutional Review Board of the New York Academy of Medicine approved study procedures. Refer to Galea et al. (2003) for additional 
information on the study design, recruitment procedures, interview content, and major findings of the larger study.

\section{RESULTS}

Cronbach's alpha was .83 for the NWS-PTSD and .90 for the PCL. Corrected item-total coefficients ranged from .18 to .55 on the NWS-PTSD $(M d n=.47)$ and from .47 to .70 on the PCL $(M d n=.60)$. Interitem correlation coefficients ranged from .04 to .47 on the NWS-PTSD and from .16 to .70 on the PCL. Independent-samples $t$ tests using the NWS-PTSD symptom counts (due to low base-rate in diagnosis) and PCL total scores revealed that these measures were similar in their relation to exposure variables. Both measures were associated with fear of injury or death at the time of the September 11 attacks, 32.8 (yes) versus 22.9 (no) on the PCL, $t(209)=6.25$, $p<.001 ; 2.4$ vs. 1.0 on the NWS-PTSD, $t(209)=3.69$, $p<.001$. Both measures were also associated with job loss as a result of the attacks, 34.8 (yes) versus 24.7 (no) on the PCL, $t(209)=2.84, p<.01 ; 3.7$ vs. 1.2 on the NWSPTSD, $t(209)=2.95, p<.01$. Neither measure was associated with respondents' witnessing of the terrorist attacks in person or whether respondents lived in close proximity to the World Trade Center site. The only exposure variable on which the measures differed from one another was death of a relative, friend, or acquaintance, which was associated with the NWS-PTSD scores, 2.1 (yes) versus 1.0 (no), $t(209)=3.18, p<.01$, but not the PCL scores, 26.7 versus $24.5, t(209)=1.43$, $n$ s.

\section{Prevalence Estimates}

For the NWS-PTSD, the prevalence of probable PTSD was calculated based on respondents' satisfying Criteria $\mathrm{B}$, $\mathrm{C}$, and D for PTSD, with the additional requirement that re-experiencing and content-specific avoidance symptoms be identified by participants as having been related to the World Trade Center disaster (these criteria also were used by Galea et al., 2002). For the PCL, probable PTSD was calculated using Weathers and colleagues' (1993) recommended cutoff of $\geq 50$ (also used by Schlenger et al., 2002).
Based on these criteria, overall prevalence of probable pastmonth PTSD was $1.7 \%$ for the NWS-PTSD and $4.1 \%$ for the PCL. This analysis yielded a kappa coefficient of .45. Although a comparable prevalence on the PCL and NWS-PTSD could be obtained using a PCL cutoff of 60 and above (prevalence $=1.8 \%$ ), kappa was particularly low (.21) at this level.

Table 1 describes prevalence estimates based on the NWS-PTSD and the PCL data at the symptom, cluster, and diagnostic levels. For chi-square analyses, Cronbach's alpha was set at .01 due to the high number of comparisons. The PCL prevalence was significantly higher than the NWS-PTSD prevalence on 16 of 17 symptoms and all three symptom clusters (i.e., re-experiencing, avoidance, hyperarousal) when a PCL cutoff of 2 was set for symptom endorsement (i.e., minimum of a little bit). Using a PCL cutoff of 3 (i.e., minimum of moderately), the PCL prevalence was higher than the NWS-PTSD prevalence on 8 of 17 symptoms as well as the re-experiencing and hyperarousal symptom clusters; the NWS-PTSD prevalence was higher on only one symptom (i.e., recall). Using a PCL cutoff of 4 for symptom endorsement (quite a bit or extremely), similar prevalences emerged at the cluster level as well as on 13 of 17 symptoms-(NWS-PTSD prevalence was higher than the PCL for two symptoms; PCL prevalence was higher than the NWS-PTSD for two symptoms). However, all of these symptom-level cutoffs, when applying the requirement of one or more re-experiencing symptom plus three or more avoidance symptoms plus two or more hyperarousal symptoms, were less concordant with the NWS-PTSD diagnoses than was a PCL cutoff of $\geq 50$ $(\kappa=.45)$.

\section{Correlates of Disagreement Between the NWS-PTSD and the PCL}

An additional series of analyses were conducted to identify circumstances under which disagreement occurred most frequently between these measures. Specifically, demographic (i.e., age, gender, racial/ethnic status, income, education level) and September 11 variables (i.e., death of 
Table 1. NWS-PTSD Versus PCL: Symptom, Cluster, and Diagnostic Prevalence

\begin{tabular}{|c|c|c|c|c|c|}
\hline Symptom descriptors & NWS- PTSD & $\begin{array}{c}\text { PCL } \\
(\operatorname{Min}=2)\end{array}$ & $\begin{array}{c}\text { PCL } \\
(\mathrm{Min}=3)\end{array}$ & $\begin{array}{c}\text { PCL } \\
(\mathrm{Min}=4)\end{array}$ & $\begin{array}{c}\text { PCL } \\
(\operatorname{Min}=5)\end{array}$ \\
\hline \multicolumn{6}{|l|}{ PTSD symptoms } \\
\hline 1. Intrusive images & 7.7 & $43.5^{\mathrm{a}}$ & $28.3^{\mathrm{a}}$ & $15.7^{\mathrm{a}}$ & 8.3 \\
\hline 2.Repeated nightmares & 3.4 & $16.8^{\mathrm{a}}$ & $11.2^{\mathrm{a}}$ & 4.7 & 2.6 \\
\hline 3.Flashback & 1.3 & $17.7^{\mathrm{a}}$ & $11.3^{\mathrm{a}}$ & 4.3 & 2.6 \\
\hline 4.Psychological reactivity & 2.1 & $47.6^{\mathrm{a}}$ & $34.2^{\mathrm{a}}$ & $18.6^{\mathrm{a}}$ & $10.0^{\mathrm{a}}$ \\
\hline 5. Physiological reactivity & 3.4 & $12.6^{\mathrm{a}}$ & 5.2 & 2.2 & 0.9 \\
\hline 6. Avoiding thoughts, feelings & 10.7 & $23.9^{\mathrm{a}}$ & 14.8 & 6.1 & $2.6^{\mathrm{b}}$ \\
\hline 7. Avoiding activities, places & 4.7 & $15.7^{\mathrm{a}}$ & 10.0 & 5.7 & 2.2 \\
\hline 8. Inability to recall & 15.9 & 9.6 & $5.7^{\mathrm{b}}$ & $3.5^{\mathrm{b}}$ & $2.6^{\mathrm{b}}$ \\
\hline 9. Lost interest in activities & 6.0 & $17.7^{\mathrm{a}}$ & $13.0^{\mathrm{a}}$ & 5.6 & 3.0 \\
\hline 10. Feelings of detachment & 6.0 & $14.3^{\mathrm{a}}$ & 9.1 & 4.8 & 2.6 \\
\hline 11. Restriction of affect & 5.2 & $12.6^{\mathrm{a}}$ & 7.0 & 4.3 & 2.6 \\
\hline 12. Foreshortened future & 3.4 & $27.5^{\mathrm{a}}$ & $16.2^{\mathrm{a}}$ & $10.0^{\mathrm{a}}$ & 2.6 \\
\hline 13. Sleep difficulties & 15.0 & $29.4^{\mathrm{a}}$ & 19.0 & 10.4 & $3.0^{\mathrm{b}}$ \\
\hline 14. Irritable, angry outbursts & 12.9 & $22.0^{\mathrm{a}}$ & 12.5 & $3.4^{\mathrm{b}}$ & $2.2^{\mathrm{b}}$ \\
\hline 15. Concentration difficulties & 4.7 & $20.7^{\mathrm{a}}$ & 8.6 & 3.4 & 1.3 \\
\hline 16. Hypervigilance & 4.7 & $39.0^{\mathrm{a}}$ & $27.2^{\mathrm{a}}$ & $14.0^{\mathrm{a}}$ & 7.0 \\
\hline 17. Exaggerated startle & 4.3 & $22.9^{\mathrm{a}}$ & $12.1^{\mathrm{a}}$ & 5.6 & 3.0 \\
\hline \multicolumn{6}{|l|}{ PTSD symptom clusters } \\
\hline Re-experiencing (Criterion B) & 11.2 & $63.8^{\mathrm{a}}$ & $43.7^{\mathrm{a}}$ & $24.5^{\mathrm{a}}$ & 14.8 \\
\hline Avoidance (Criterion C) & 5.2 & $17.8^{\mathrm{a}}$ & $11.1^{\mathrm{a}}$ & 5.8 & 3.6 \\
\hline Hyperarousal (Criterion D) & 9.0 & $36.1^{\mathrm{a}}$ & $21.1^{\mathrm{a}}$ & 9.3 & 3.5 \\
\hline PTSD Criteria B, C, and D & 1.7 & $13.9^{\mathrm{a}}$ & $7.4^{\mathrm{a}}$ & 3.0 & 1.7 \\
\hline
\end{tabular}

a friend, relative, or acquaintance; loss of job; saw terrorist attacks in person; fear of injury or death; resided in close proximity to World Trade Center site) were examined in relation to symptom and diagnostic-level disagreement on the NWS-PTSD and the PCL. Chi-square analyses were conducted at the symptom cluster level, where one or more symptom was required to meet the re-experiencing criterion, three or more symptoms for the avoidance criterion, and two or more symptoms for the hyperarousal criterion. A PCL cutoff of 4 was used for symptom endorsement because this level most closely approximated the NWSPTSD prevalence estimates as described above and shown in Table 1. Next, chi-square analyses were conducted to examine disagreement at the diagnostic level (i.e., the NWS-
PTSD diagnosis against the widely used PCL cutoff score of 50). Due to the number of analyses (i.e., 10 with each of the 4 sets of analysis), Cronbach's alpha was again set at .01.

Demographic variables were unrelated to symptom disagreement on the NWS-PTSD and PCL, with one exception: Low income was associated with less agreement on the hyperarousal criterion, $\chi^{2}(6, N=175)=16.9, p<.01$. Participants with incomes of $\$ 75,000$ or more gave concordant responses $96.1 \%$ of the time versus $65.7 \%$ for participants with incomes of less than $\$ 20,000$. No pattern was evident in nonconcordant responses: 15 participants met the hyperarousal criterion on the NWSPTSD but not PCL, whereas 13 met this criterion on the PCL but not NWS-PTSD. With regard to September 11 
characteristics, three variables were unrelated to symptom disagreement on the NWS-PTSD and PCL: death of a relative, friend, or acquaintance; whether or not participants saw the attacks in person; and proximity of residence to the World Trade Center site. However, two September 11 variables were associated with disagreement. First, loss of one's job due to the attacks was associated with less agreement on the avoidance criterion (66.7 vs. $93.0 \%), \chi^{2}(2$, $N=224)=10.7, p<.01$, as well as the hyperarousal criterion (55.6 vs. $87.1 \%), \chi^{2}(2, N=226)=10.3, p<.01$. For both of these analyses, relatively equal numbers of participants met criteria on the NWS-PTSD but not the PCL as had met on the PCL but not the NWS-PTSD. Second, fear of injury death at the time of the terrorist attacks was associated with less agreement on all three PTSD criteria: re-experiencing (63.5 vs. $81.4 \%), \chi^{2}(2$, $N=224)=11.2, p<.01$; avoidance (82.7 vs. $94.7 \%)$, $\chi^{2}(2, N=222)=9.0, p=.01$; and hyperarousal $(73.1$ vs. $90.1 \%), \chi^{2}(2, N=223)=10.7, p<.01$. No clear pattern of disagreement emerged relative to the avoidance and hyperarousal criteria, but for the re-experiencing criterion most participants with nonconcordant responses (39 of $51 ; 76.5 \%)$ met this criterion on the PCL but not on the NWS-PTSD.

Analyses with diagnostic-level outcomes should be considered exploratory and interpreted with caution because of the particularly low base-rate of disagreement (i.e., 7/221, $3.2 \%$ valid cases) at this level. Disagreement between the NWS-PTSD and PCL measures at the diagnostic level was statistically unrelated to all demographics examined: age, gender, racial/ethnic status, income level, and educational status. Disagreement was also unrelated to death of a friend, relative, or acquaintance due to the September 11 attacks; loss of one's job; whether or not participants saw the attacks in person; and proximity of residence to the World Trade Center site. However, participants' reported fear of injury or death at the time of the September 11 attacks was associated with disagreement. Specifically, disagreement between the NWS-PTSD and the PCL was more likely to occur when peritraumatic fear was endorsed $(9.6 \%)$ versus denied (1.2\%), $\chi^{2}(2, N=218)=12.3, p<.01$. In all five instances of disagreement among participants who en- dorsed peritraumatic fear, a PCL score of 50 or higher had been obtained without satisfying diagnostic criteria based on the NWS-PTSD measure.

\section{DISCUSSION}

The prevalence of probable past-month PTSD in this sample was 1.7 and $4.1 \%$ for the NWS-PTSD and the PCL, respectively, based on interviews conducted 4-5 months following the terrorist attacks of September 11, 2001. Although the nature of the sample and low base-rate of PTSD limit our ability to draw definitive conclusions regarding the degree to which these instruments differ in their estimation of population-based prevalence, the current findings suggest that the PCL tends to yield higher prevalences at the symptom, cluster, and diagnostic levels. As described in Table 1, these measures performed similarly with regard to prevalence at the symptom and cluster levels when the cutoff for symptom endorsements on the PCL was set at 4 (quite a bit or extremely). However, kappa coefficients between the measures were highest when a PCL cutoff score of 50 was used. This finding supports the suggestion of Weathers and colleagues (1993) to use this cut-point when generating estimates of probable PTSD with the PCL.

This pattern of findings is consistent with that of Galea et al. (2002) and Schlenger et al. (2002) in their assessment of probable PTSD among New York City-area residents following the September 11 attacks. Both studies estimated prevalence in the 1-2 months following the attacks, and both studies assessed symptoms using the same time frame (i.e., past month) with large, representative samples. The sampling frame for Galea et al. was restricted to households south of 110th Street on Manhattan (in close proximity to the World Trade Center), whereas Schlenger et al. sampled more broadly from the New York City metropolitan area. Yet, despite recruiting a sample with more direct exposure to the September 11 attacks, Galea et al. reported a prevalence of probable past-month PTSD of 7.5\%, 50\% lower than the prevalence of $11.2 \%$ reported by Schlenger and colleagues. Consistent with findings of the present study, it is likely that the selection of PTSD instruments 
contributed to this difference in prevalence estimates (as noted earlier, Galea et al. administered the NWS-PTSD, whereas Schlenger et al. administered the PCL).

Results of the current study shed light on the circumstances under which symptom disagreement occurs on the NWS-PTSD versus the PCL. However, although several predictors of disagreement were identified (i.e., income, loss of job, fear of injury or death), few clear patterns emerged in the data that favored either instrument. It will be important for future research to examine correlates of disagreement in greater depth using samples that provide increased statistical power via higher base-rates of PTSD. Findings from this study also bring us a step closer toward enhancing the comparability of the numerous epidemiological studies that have used the PCL or the NWS-PTSD to generate population-based prevalence estimates. However, these data cannot speak to the relative diagnostic accuracy and precision of these measures; that is, they do not address whether the NWS-PTSD yields underestimates versus the PCL yielding overestimates of prevalence. It is likely that differences in prevalence estimates are accounted for by structural dissimilarities between these measures, such as the use of a Likert scale versus yes/no response options, or the assessment of symptom severity or distress (PCL) versus symptom occurrence or frequency (the NWS-PTSD). For example, an individual may experience only one nightmare in a given week, but if the nightmare is particularly distressing the individual may have difficulty managing their emotional reactions and may be inclined to report that they were extremely bothered by the symptom. Alternatively, an individual may experience significant physical reactivity to victimization-related cues on a very frequent basis, but if they come to accept these reactions and do not adjust their lifestyle to avoid them, they may be inclined to report that they are minimally bothered by the symptom.

Given that the NWS-PTSD and the PCL differ somewhat in their structure and detection of symptoms and therefore may tap into different aspects of symptom presentation, it may be that they are better suited for use in different contexts. In light of the lack of data that speak to this issue, some additional structural observations are worth noting. Based strictly on structural comparisons between the measures, one of the benefits for epidemiological research of the NWS-PTSD is its direct modeling from $D S M-I V$ criteria and dichotomous (yes/no) response options that allow for estimates of PTSD diagnosis based strictly on DSM criteria. Criteria are less directly DSMbased on the PCL because cutoff scores are used rather than traditional DSM requirements of one re-experiencing, two hyperarousal, and three avoidance symptoms (also see Forbes, Creamer, \& Biddle, 2001). Further, although the PCL cutoff score of 50 has generally fared well with regard to diagnostic sensitivity and specificity, recommended cutoff scores for diagnosis have varied across populations of individuals exposed to traumatic stressors: 30 for breast cancer patients (e.g., Andrykowski, Cordova, Studts, \& Miller, 1998), 44 with sexual assault and motor vehicle accident victims (e.g., Blanchard et al., 1996), and 50 with combat veterans and disaster-affected civilian samples (e.g., Weathers et al., 1993). This suggests that the commonly recommended PCL cutoff of 50 is optimal only for certain populations, consistent with results of Andrykowski et al. (1998) who reported a diagnostic sensitivity of only .60 (specificity was .99) using a PCL cut-point of 50 with their sample of breast cancer patients. For these reasons, it is possible that the NWS-PTSD is better suited for epidemiological studies that focus specifically on diagnostic prevalence. However, it is premature to conclude this with confidence until sufficient research has addressed this issue. On the other hand, the PCL may be better equipped than the NWS-PTSD to detect symptom change in clinical practice or treatment outcome research given its wider range for symptom response options using a Likert scale format and total scores. The PCL produces scores with a possible range of 17 to 85 , whereas the NWS-PTSD measure produces symptom counts with a possible range of 0 to 17 . This structural difference allows for higher variability in PCL scores and therefore may strengthen statistical power to detect symptom change over time. Forbes et al. (2001) noted, however, that the PCL may underestimate pretreatment to posttreatment improvements in symptoms relative to the Clinician-Administered PTSD scale (Blake, Weathers, Nagy, Kaloupek, Klauminzer, Charney, et al., 1990). Thus, 
the strengths and limitations of both measures require further investigation to inform their use in epidemiologic and clinical research.

Future research should address the advantages and disadvantages associated with structural differences of PTSD measurement scales. The paucity of research on this issue is surprising considering the availability of several widely used measures of PTSD that assess symptom frequency (e.g., the NWS-PTSD, the Trauma Symptom Inventory, (Brierre, 1995.) the UCLA PTSD Index,) (Pynoos, Rodriguez, Steinberg, Stuber, Frederick, 1998), and several others that assess symptom intensity or associated distress (e.g., the PCL, the Impact of Event Scale-Revised (Weiss and Marmar, 1997); the Clinician Administered PTSD Scale (Blake et al., 1990) assesses both frequency and intensity). Measures that differ in their approach to assessing symptoms may be capturing somewhat different dimensions of PTSD that, in turn, may relate in distinct ways to the conditioning and extinction of fearful responding as well as the stability of emotional functioning over time. Using a sample with a higher PTSD base rate to examine differences and patterns of disagreement in greater depth at the item level may assist in answering some of these questions.

Considerable variability in prevalence estimates in national and community studies has long been recognized in epidemiological research, but little is known about the extent to which measurement differences account for this. Thus, on a broad scale, greater research attention is needed on the similarities and differences, both structural and functional, between widely used mental health and physical-health-related measures in epidemiological research. This study illustrates, through comparison of two commonly used instruments, the implications of instrument selection. Clearly, interpretation of epidemiologic data obtained with different screening measures needs to take into account the implications of measure selection. The field would also benefit from research that examines measurement structure relative to relations between symptoms and functional impairment. As greater awareness is achieved of the similarities and differences between widely used measures, researchers and policymakers may have an improved ability to account for this variability with improved clarity and precision.

\section{REFERENCES}

American Psychiatric Association. (1994). Diagnostic and statistical manual of mental disorders (4th ed.). Washington, DC: Author.

Andrykowski, M. A., Cordova, M. J., Studts, J. L., \& Miller, T. W. (1998). Posttraumatic stress disorder after treatment for breast cancer: Prevalence of diagnosis and use of the PTSD ChecklistCivilian Version (PCL-C) as a screening instrument. Journal of Consulting and Clinical Psychology, 66, 586-590.

Blake, D., Weathers, F., Nagy, L., Kaloupek, D., Klauminzer, G., Charney, D., et al. (1990). A clinician rating scale for assessing curent and lifetime PSTD: The CAPS-1. The Behavior Therapist, 18, 187-188.

Blanchard, E. B., Jones-Alexander, J., Buckley, T. C., \& Forneris, C. A. (1996). Psychometric properties of the PTSD Checklist (PCL). Behaviour Research and Therapy, 34, 669-673.

Brierre, J. (1995). Trauma Symptom Inventory (TSI): Professional manual. Odessa, FL: Psychological Assessment Resources.

Forbes, D., Creamer, M., \& Biddle, D. (2001). The validity of the PTSD checklist as a measure of symptomatic change in combatrelated PTSD. Behaviour Research and Therapy, 39, 977986.

Galea, S., Ahern, J., Resnick, H., Kilpatrick, D., Bucuvalas, M., Gold, J., et al. (2002). Psychological sequelae of the September 11 terrorist attacks in New York City. New England Journal of Medicine, 346, 982-987.

Galea, S., Nandi, A., \& Vlahov, D. (2005). The epidemiology of post-traumatic stress disorder after disasters. Epidemiologic Reviews, 27, 78-91.

Galea, S., Vlahov, D., Resnick, H., Ahern, J., Susser, E., Gold, J., et al. (2003). Trends of probable post-traumatic stress disorder in New York City after the September 11 terrorist attacks. American Journal of Epidemiology, 158, 514-524.

Garrison, C. Z., Bryant, E. S., Addy, C. L., Spurrier, P. G., Freedy, J. R., \& Kilpatrick, D. G. (1995). Posttraumatic stress disorder in adolescents after Hurricane Andrew. Journal of the American Academy of Child and Adolescent Psychiatry, 34, 1193-1201.

Hanson, R. F., Kilpatrick, D. G., Freedy, J. R., \& Saunders, B. E. (1995). Los Angeles County after the 1992 civil disturbances: Degree of exposure and impact on mental health. Journal of Consulting and Clinical Psychology, 63, 987-996. 
Hoge, C. W., Castro, C. A., Messer, S. C., McGurk, D., Cotting, D. I., \& Koffman, R. L. (2004). Combat duty in Iraq and Afghanistan, mental health problems, and barriers to care. New England Journal of Medicine, 351, 13-22.

Iowa Persian Gulf Study Group. (1997). Self-reported illness and health status among Gulf War veterans: A population-based study. Journal of the American Medical Association, 277, 238245.

Kessler, R. C., Sonnega, A., Bromet, E., Hughes, M., \& Nelson, C. (1995). Posttraumatic stress disorder in the National Comorbidity Survey. Archives of General Psychiatry, 52, 1048-1060.

Kilpatrick, D. G., Resnick, H. S., Freedy, J. R., Pelcovitz, D., Resick, P. A., Roth, S., et al. (1998). The posttraumatic stress disorder field trial: Evaluation of the PTSD construct: Criteria A through E. In T. Widiger et al. (Eds.), DSM-IV sourcebook (pp. 803-844). Washington, DC: American Psychiatric Press.

Kilpatrick, D. G., Resnick, H. S., Freedy, J. R., Pelcovitz, D., Resnick, P. A., Roth, S., et al. (1998). The posttraumatic stress disorder field trial: Evaluation of the PSTD construct - Criteria A through E. In Widiger, H., Pincus, R. Ross, First, W. Davis, et al. (Eds.), DSM-IV sourcebook (Vol. 4, pp. 803-844). Washington, DC: American Psychiatric Press.

Kilpatrick, D. G., Resnick, H. S., Saunders, B. E., \& Best, C. L. (1989). The National Women's Study PTSD Module. Charleston, SC: Medical University of South Carolina, Department of Psychiatry \& Behavioral Sciences.

Kilpatrick, D. G., Ruggiero, K. J., Acierno, R. E., Saunders, B. E., Resnick, H. S., \& Best, C. L. (2003). Violence and risk of PTSD, major depression, substance abuse/dependence, and comorbidity: Results from the National Survey of Adolescents. Journal of Consulting and Clinical Psychology, 71, 692700 .
Kulka, R. A., Schlenger, W. E., Fairbank, J. A., Hough, R. L., Jordan, B. K., Marmar, C. R., et al. (1990). Trauma and the Vietnam War generation: Report of findings from the National Vietnam Veterans Readjustment Study. New York: Brunner/Mazel.

Pynoos, R., Rodriguez, N., Steinberg, A., Stuber, M., \& Frederick, C. (1998). UCLA PTSD Index for DSM-IV: Child, Adolescent, and Parent versions. UCLA Trauma Psychiatry Service.

Resnick, H. S., Kilpatrick, D. G., Dansky, B. S., Saunders, B. E., \& Best, C. L. (1993). Prevalence of civilian trauma and posttraumatic stress disorder in a representative national sample of women. Journal of Consulting and Clinical Psychology, 61, 984991.

Ruggiero, K. J., Del Ben, K., Scotti, J., \& Rabalais, A. (2003). Psychometric properties of the PTSD Checklist-Civilian Version. Journal of Traumatic Stress, 16, 495-502.

Schlenger, W. E., Caddell, J. M., Ebert, L., Jordan, B. K., Rourke, K. M., Wilson, D., et al. (2002). Psychological reactions to terrorist attacks: Findings from the National Study of Americans' Reactions to September 11. Journal of the American Medical Association, 288, 581588.

Schuster, M. A., Stein, B. D., Jaycox, L. H., Collins, R. L., Marshall, G. N., Elliott, M. N., et al. (2001). A national survey of stress reactions after the September 11, 2001, terrorist attacks. New England Journal of Medicine, 345, 1507-1512.

Spitzer, R. L., Williams, J. B. W., and Gibbon, M. (1986). Structured clinical interview for DSM-III-R, nonpatient version. New York State Psychiatric Institute, NY: Biometrics Research Department.

Weathers, F. W., Litz, B. T., Herman, D. S., Huska, J. A., \& Keane, T. M. (1993, October). The PTSD Checklist (PCL): Reliability, validity, and diagnostic utility. Paper presented at the Meeting of the International Society for Traumatic Stress Studies, San Antonio, TX. 